\title{
HYPERPLANE SECTIONS OF CALABI-YAU VARIETIES
}

\author{
JONATHAN WAHL \\ In Memory of My Friend Michael Schneider
}

\begin{abstract}
Theorem. If $W$ is a smooth complex projective variety with $h^{1}\left(\mathcal{O}_{W}\right)=$ 0 , then a sufficiently ample smooth divisor $X$ on $W$ cannot be a hyperplane section of a Calabi-Yau variety, unless $W$ is itself a Calabi-Yau.

Corollary. A smooth hypersurface of degree $d$ in $\mathbb{P}^{n}(n \geq 2)$ is a hyperplane section of a Calabi-Yau variety iff $n+2 \leq d \leq 2 n+2$.

The method is to construct out of the variety $W$ a universal family of all varieties $Z$ for which $X$ is a hyperplane section with normal bundle $K_{X}$, and examine the "bad" singularities of such $Z$.

It was proved in [W1] that if a smooth curve lies on a $K-3$ surface, its GaussianWahl map $\Phi_{K}$ is not surjective.

Theorem. The following smooth curves do not lie on a $K$-3, even though $\Phi_{K}$ is not surjective: plane curves of degree $\geq 7$; bielliptic curves of genus $\geq 11$; curves on $\mathbb{F}_{n}$ of degree $\geq 5$ over $\mathbb{P}^{1}$.
\end{abstract}

1991 Mathematics Subject Classification. AMS Subject Classification Numbers 14J32, 14 D15. 


\section{INTRODUCTION}

A Calabi-Yau variety shall mean a complex projective variety $Y$ with trivial dualizing sheaf $K_{Y} \cong \mathcal{O}_{Y}$ and $h^{1}\left(\mathcal{O}_{Y}\right)=0$, with only isolated canonical (i.e. rational) singularities. When $\operatorname{dim} Y=2, Y$ is a $K-3$ surface, possibly with rational double points. If $X$ is a smooth hyperplane section of a Calabi-Yau, by the adjunction formula $K_{X}$ is very ample; if $\operatorname{dim} Y \geq 3$ also $h^{1}\left(\mathcal{O}_{X}\right)=0$.

Conversely, given a canonically polarized $\left(X, K_{X}\right)$, one asks if it can be a hyperplane section of a Calabi-Yau. Expected finiteness results for families of Calabi-Yaus suggest such $\left(X, K_{X}\right)$ should be quite special. For instance, there is a 19-dimensional family of $K-3$ surfaces with a hyperplane section of genus $g$, hence only a $(g+19)$ dimensional family of such curves [MoM]. Still, $K-3$ curves can be Brill-Noether-Petri general [L].

By considering deformations of the affine cone $A$ over a canonical curve, we showed in [W1] that a curve $X$ on a $K-3$ has non-surjective Gaussian-Wahl map $\Phi_{K}: \Lambda^{2}(K) \rightarrow \Gamma\left(K^{\otimes 3}\right)$; the relevant part of the tangent space to the deformations of $A$ must be non-0. Curves with surjective $\Phi_{K}$ include most complete intersections and the generic curve of genus 10 or $\geq 12[\mathrm{CHM}]$; such $X$ could not be on a $K-3$. Conversely, it is conjectured in [W2] that a Brill-Noether-Petri general curve with $\Phi_{K}$ non-surjective does indeed lie on a $K-3$.

This paper shows many $X$ are not hyperplane sections of a Calabi-Yau, even when there are "interesting" deformations of the cone. For instance, smooth plane curves of degree $\geq 7$ cannot lie on a $K-3$ [GL], although $\Phi_{K}$ has corank 10 . For $\operatorname{dim} X \geq 2$, the relevant tangent space is $H^{1}\left(X, \Theta_{X} \otimes K_{X}^{-1}\right)$, which is always non-0 for $X \subset \mathbb{P}^{n}$ a smooth hypersurface; but we prove that for degree $>2 n+2$, such an $X$ can not lie on a Calabi-Yau. Our method is to describe explicitly all deformations of the cone.

We actually adopt the point of view of extensions of a subvariety $V \subset \mathbb{P}^{n}$ rather than deformations of the cone: consider $W \subset \mathbb{P}^{n+k}$ for which a codimension $k$ transversal linear section gives $V$. Any projective variety is an extension of its hyperplane section. There is the notion of a "universal extension" of a given $V$ which in good cases is governed by a Kodaira-Spencer map. Combining Theorem 2.8 with Remark 1.10.1 gives

Theorem A. Suppose $V \subset \mathbb{P}^{n}$ satisfies $\left(N_{2}\right), V \nsubseteq \mathbb{P}^{1}$, and $W \subset \mathbb{P}^{n+k}$ is an extension. From the normal bundle sequence of $V$ in $W$ consider the coboundary map

$$
\gamma: \Gamma\left(N_{V / W}(-1)\right) \rightarrow H^{1}\left(\Theta_{V}(-1)\right) .
$$

Assume either

(i) $\operatorname{dim} V=1$ and $\operatorname{crk} \gamma=\operatorname{crk} \Phi\left(K_{V}, \mathcal{O}_{V}(1)\right)$ ( $\Phi$ a Gaussian)

(ii) $\operatorname{dim} V \geq 2$ and $\gamma$ is an isomorphism.

Then $W$ is a universal extension of $V$, in an appropriate sense. 
As remarked in (1.10.4), this result may be used to deduce Mukai's classification of Gorenstein Fano 3-folds of genus 7, 8, or 9 from his earlier results on curves [M2]. In $[\mathrm{BM}]$, it is shown that for a hyperplane section of a $K-3$ surface, $\gamma \neq 0$ (i.e., the normal sequence is non-split) unless there is an involution fixing the section.

Next we give a general method for constructing extensions of a canonically polarized $\left(X, K_{X}\right)$, in case $X$ is a divisor on a variety $Z$ (usually Fano). We attribute this method to DuVal [D], though a similar use of adjoint linear systems goes back to del Pezzo. We illustrate by considering a plane curve $C$ of degree $d \geq 4$ (cf. [E], [W2]). Let $D$ be a smooth cubic intersecting $C$ transversally, with $Z=C \cap D$. The linear system of curves of degree $d$ containing $Z$ defines a morphism on the blow-up $B \rightarrow \mathbb{P}^{2}$ of $Z$; this restricts to the canonical embedding of the proper transform of $C$, and is an embedding except for collapsing the proper transform of $D$ to a point. The image of $B$ is a normal surface with trivial dualizing sheaf and one simple elliptic singularity, for which $C$ is a hyperplane section. If $d \geq 7$, keeping track of the normal sequence one can show all extensions of $C \subset \mathbb{P}^{g-1}$ arise from varying $D \in\left|-K_{\mathbb{P}^{2}}\right|$, with possibly worse (hence still non-rational) singularities. Since no such is a $K-3, C$ lies on no $K-3$.

This method generalizes greatly to large divisors on an arbitrary $X$ (Corollary 3.12); one knows all extensions of the canonical embedding. Combining (3.12) and (3.13) yields the chief result of this paper:

Theorem B. Let $Z$ be a projective variety with isolated Gorenstein singularities and $h^{1}\left(\mathcal{O}_{Z}\right)=0$. Then for sufficiently ample smooth divisors $X$ on $Z, X$ does not sit on a Calabi-Yau, unless $Z$ is already a Calabi-Yau.

There is a much sharper statement when $\operatorname{dim} Z=2$ (Theorem 4.5). We also remark that Theorem $\mathrm{B}$ is fairly easy in case $-K_{Z}$ has no global sections; in that case the only extensions of $X$ are cones.

Corollary 4.5: A smooth hypersurface of degree $d$ in $\mathbb{P}^{n}$ is a hyperplane section of a Calabi-Yau variety iff $n+1 \leq d \leq 2 n+2$.

In $\S 1$, we introduce the (first) Kodaira-Spencer map of an extension and prove (Theorem 1.9) that it can be used to prove a given extension is universal. We relate this map to the normal bundle sequence of an extension in $\S 2$. In $\S 3$, start with $X \subset Y$ a divisor, with $h^{1}\left(\mathcal{O}_{Y}\right)=0$; for an effective divisor $D \subset Y$, we construct an extension of $X$ embedded via $\mathcal{O}_{Y}(X-D) \otimes \mathcal{O}_{X}$, by using the rational map of $Y$ given by sections of $\Gamma\left(Y, \mathcal{I}_{X \cap D}(X)\right)$. One can do this as well for a family of $D$ 's; using $\S 2$, one has criteria that a universal extension has been constructed. These results are applied to canonical embeddings of curves in Theorem 4.5, yielding the universal extension for all complete intersection curves, most curves on the Hirzebruch surfaces $\mathbb{F}_{n}$, and bielliptic curves of genus $\geq 11$. In particular, we find that only the "obvious" complete intersection curves actually lie on a $K-3$. Finally, in $\S 5$, we give 
some necessary conditions for a smooth complete intersection in $\mathbb{P}^{n}$ of dimension at least 2 to be a hyperplane section of a Calabi-Yau.

It should be clear that a more accurate (though much more cumbersome) title for this paper would have been "Varieties which are not hyperplane sections of CalabiYau manifolds".

This research was partially supported by an NSF Grant.

\section{Extensions of SUbVARIETIES}

(1.1) Let $V \subset \mathbb{P}^{n}$ be a non-degenerate subvariety. Identify $\mathbb{P}^{n}$ with a linear subspace $H$ of $\mathbb{P}^{n+k}$. A (k-step) extension of $V$ is a subvariety $W$ of $\mathbb{P}^{n+k}$ so that $V=W \cap H$, transversally; thus, $\operatorname{codim}(V, W)=k$, and the defining equations of $H$ form locally a regular sequence in $W\left(\mathrm{cf}\right.$. [Lv]). Two extensions $\left(V, H, W, \mathbb{P}^{r}\right)$ and $\left(V, H^{\prime}, W^{\prime}, \mathbb{P}^{r}\right)$ are isomorphic if there is an isomorphism of $\mathbb{P}^{r}$ sending $H$ onto $H^{\prime}$, $W$ onto $W^{\prime}$, and equal to the identity on $V$; in particular, extensions $W$ and $W^{\prime}$ of $V \subset H \subset \mathbb{P}^{n+k}$ are isomorphic if they differ by an automorphism of $\mathbb{P}^{n+k}$ which is the identity on $H$. From $W \subset \mathbb{P}^{n+k}$, one may form a sub-extension $(V, H, W \cap L, L)$, where $H \subset L \subset \mathbb{P}^{n+k}$, as well as a cone over $W$ in $\mathbb{P}^{n+k+1}$. When $V \subset \mathbb{P}^{n}$ is linearly normal, one may speak of an extension of $\left(V, \mathcal{O}_{V}(1)\right)$.

(1.2) Consider $V \subset H \subset \mathbb{P}^{n+1}$, and a projective cone $C(V)$ of $V$ over a point in $\mathbb{P}^{n+1}-H$; so $C(V) \cap H=V$. There is a relation between extensions of $V \subset H$; part of the Hilbert scheme of $C(V) \subset \mathbb{P}^{n+1}$; and deformations of non-positive weight of the affine cone of $V$ in $\mathbb{C}^{n+1}$. For instance, suppose $W$ is a fibre in a deformation of $C(V)$ in $\mathbb{P}^{n+1}$ which keeps fixed a hyperplane section: $W \cap H=C(V) \cap H=V$; then $\left(V, H, W, \mathbb{P}^{n+1}\right)$ is an extension of $V$. Conversely, if $\left(V, H, W, \mathbb{P}^{n+1}\right)$ is a 1-step extension, then by a well-known construction (e.g., [P]) $W$ degenerates in $\mathbb{P}^{n+1}$ to a projective cone over a hyperplane section $W \cap H=V$. On the other hand, it is a bit tricky to construct appropriate deformation theories from the point of view of the Hilbert scheme with fixed hyperplane section, or for the negative weight deformations of the affine cone.

(1.3) A $k$-step extension $W$ of $V$ is universal if every extension of $V$ is equivalent to a (possibly trivial) projective cone over a unique subextension. Such a $W$ has the weaker property (cf. $[\mathrm{Lv}]$ ) that any extension of it is a cone. A universal extension need not exist. We give a criterion for a given extension of $V$ to be universal, using a Kodaira-Spencer map.

(1.4) For $\left(V, H, W, \mathbb{P}=\mathbb{P}^{n+k}\right)$ a $k$-step extension, there is a natural KodairaSpencer map

$$
K S: \Gamma\left(\mathbb{P}, \mathcal{I}_{H}(1)\right)^{*} \rightarrow \Gamma\left(V, N_{V / H}(-1)\right) / \Gamma\left(H, \Theta_{H}(-1)\right) .
$$

Here, $\mathcal{I}_{H}$ is the ideal sheaf of $H$ in $\mathbb{P}$ and $N$ represents the normal sheaf. For, use the short exact sequence

$$
0 \rightarrow \Gamma\left(\mathcal{O}_{H}(1)\right)^{*} \rightarrow \Gamma\left(\mathcal{O}_{\mathbb{P}}(1)\right)^{*} \rightarrow \Gamma\left(\mathcal{I}_{H}(1)\right)^{*} \rightarrow 0
$$


the sequence of maps

$$
\Gamma\left(\mathcal{O}_{\mathbb{P}}(1)\right)^{*} \cong \Gamma\left(\Theta_{\mathbb{P}}(-1)\right) \rightarrow \Gamma\left(\Theta_{\mathbb{P} \mid W}(-1)\right) \rightarrow \Gamma\left(N_{W / \mathbb{P}}(-1)\right) \rightarrow \Gamma\left(N_{V / H}(-1)\right),
$$

and the isomorphism

$$
\Gamma\left(\mathcal{O}_{H}(1)\right)^{*} \cong \Gamma\left(\Theta_{H}(-1)\right)
$$

(The map $N_{W / \mathbb{P}} \rightarrow N_{V / H}$ arises from $\mathcal{I}_{W / \mathbb{P}} \otimes \mathcal{O}_{H} \cong \mathcal{I}_{V / H}$ because the equations of $H$ give a regular sequence in $W$.) The $K S$ map of a sub-extension factors via the natural inclusion

$$
\Gamma\left(L, \mathcal{I}_{H / L}(1)\right)^{*} \subset \Gamma\left(\mathbb{P}, \mathcal{I}_{H / \mathbb{P}}(1)\right)^{*} .
$$

One can define higher-order Kodaira-Spencer maps. For example, writing $K S_{1}$ for the map above, one may consider

$$
K S_{2}: \operatorname{Ker} K S_{1} \rightarrow \Gamma\left(N_{V / H}(-2)\right),
$$

arising from considering the kernel of

$$
N_{W / \mathbb{P}}(-1) \rightarrow N_{V / H}(-1) .
$$

\section{Examples.}

(1.5.1). Suppose $Z \subset \mathbb{P}^{n}=H$ is a smooth non-degenerate subvariety, and $V=Z \cap Q$ is the transversal intersection with a quadric hypersurface. Choose coordinates $x_{0}, \cdots, x_{n}, t$ on $\mathbb{P}^{n+1}$ so that $H=\{t=0\}$. Let $W \subset \mathbb{P}^{n+1}$ be the intersection of the projective cone over $Z$ with the quadric $\left\{t^{2}=q\left(x_{0}, \cdots, x_{n}\right)\right\}$, where $q$ defines $Q$. Then $W$ is a 1-step extension of $V$; the Kodaira-Spencer map $K S$ is $0 ; \Gamma\left(N_{V / H}(-2)\right) \neq 0$; and the higher-order map

$$
\text { Ker } K S \rightarrow \Gamma\left(N_{V / H}(-2)\right)
$$

is injective.

(1.5.2). Let $V=\left\{F\left(x_{0}, \cdots, x_{n}\right)=0\right\} \subset H=\mathbb{P}^{n}$ be a non-singular hypersurface of degree $d$. Let $M_{1}, \cdots, M_{N}$ be a set of monomials giving a basis of the polynomials of degree $<d$ in $\mathbb{C}\left[x_{0}, \cdots, x_{n}\right] /\left(\frac{\partial F}{\partial x_{i}}\right)$. Let $d_{i}=\operatorname{deg} M_{i}$. Then in $\mathbb{P}^{n+N}$, with coordinates $x_{0}, \cdots, x_{n}, t_{1}, \cdots, t_{N}$, a universal extension $V$ is given by

$$
\left\{F+\sum t_{i}^{d-d_{i}} M_{i}=0\right\}
$$

In this case, the Kodaira-Spencer maps are surjections, including the $d-1$ higherorder ones, with target spaces $\Gamma\left(N_{V / H}(-i)\right), i=2, \cdots, d$.

(1.6) It will simplify matters to work with equations defining a variety, so we will assume from now on that

$V \subset H=\mathbb{P}^{n}$ is normal and projectively normal, with homogeneous coordinate ring $A=P / I(V)=P / I$. 
This assumption guarantees that for an extension $W$ of $V$, elements of $I(V)$ lift to $I(W)$. Further, the target spaces of the Kodaira-Spencer maps are identified from the following well-known facts:

(1.6.1). For all $i, \Gamma\left(V, N_{V / H}(i)\right)=i^{\text {th }}$ graded piece of $\operatorname{Hom}_{P / I}\left(I / I^{2}, P / I\right)$.

(1.6.2). $\Gamma\left(\Theta_{H}(-1)\right) \rightarrow \Gamma\left(N_{V / H}(-1)\right)$ is given by differentiation.

(1.7) Now suppose $V \subset H \subset \mathbb{P}^{n+k}=\mathbb{P}$, and choose coordinates $x_{0}, x_{1}, \cdots, x_{n}$, $t_{1}, t_{2}, \cdots, t_{k}$ so that $H$ is defined by $\left\{t_{1}=\cdots=t_{k}=0\right\}$. By $(*)$, a $k$-step extension $W \subset \mathbb{P}$ of $V$ gives a flat lifting of $I$ to $I(W) \subset \mathbb{C}\left[x_{0}, \cdots, x_{n}, t_{1}, \cdots, t_{k}\right]$. The $K S$ map of $W$ maps $\partial / \partial t_{i}$ in $\Gamma\left(\mathcal{I}_{H}(1)\right)^{*}$ to a degree -1 homomorphism $I \rightarrow P / I$ (1.6.1): if $f \in I$, lift to an $F \in I(W)$, and map to the image $\left(\partial F / \partial t_{i}\right) \equiv g_{i}$ in $P / I$. A coordinate change $x_{\gamma} \mapsto x_{\gamma}+\sum a_{\gamma \delta} t_{\delta}(\gamma=0, \cdots, n ; \delta=1, \cdots, k)$ replaces $g_{i}$ by $g_{i}+\left(\sum a_{\delta i} \partial / \partial x_{\gamma}\right) f$. So $K S\left(\partial / \partial t_{i}\right)=\left[g_{i}\right]$ in $\Gamma\left(N_{V}(-1)\right) / \Gamma\left(\Theta_{H}(-1)\right)$. (Thus $K S$ is essentially the Kodaira-Spencer map of a weight -1 deformation of the affine cone $A=P / I$.) Higher-order Kodaira-Spencer maps will involve higher-order terms in variables $t_{i}$ in Ker $K S$.

(1.8) The following ought to be well known.

Theorem 1.9. Let $V \subset \mathbb{P}^{n}=H$ be a normal and projectively normal subvariety, which satisfies

$$
H^{0}\left(V, N_{V / H}(-2)\right)=0 .
$$

Then

(a) A k-step extension $W \subset \mathbb{P}^{n+k}$ is uniquely determined (up to equivalence) by its Kodaira-Spencer map.

(b) A k-step extension $W$ with $K S$ an isomorphism is a universal extension, in that every extension is isomorphic to a (cone over a) unique subextension of $W$.

Proof. Choose coordinates as in (1.7). Encode generators of $I(V)$ in a $1 \times \kappa$ row vector $f$. Let $r$ be a $\kappa \times \ell$ matrix of forms of $P$ whose columns generate the module of relations for $f$.

$$
\begin{aligned}
& f \cdot r=0 \\
& f \cdot r^{\prime}=0, \text { where } r^{\prime} \text { is } \kappa \times s, \text { implies } r^{\prime}=r \cdot b, \text { where } b \text { is } \ell \times s .
\end{aligned}
$$

Let $W \subset \mathbb{P}^{n+k}$ be an extension of $V$. $W$ is defined by equations $F=f+\sum t^{I} g^{I}$, where $I=\left(i_{1}, \cdots, i_{k}\right),|I|=i_{1}+\cdots+i_{k}$, and $t^{I}=t_{1}^{i_{1}} t_{2}^{i_{2}} \cdots t_{k}^{i_{k}}$; relations are determined by $R=r+\sum t^{I} u^{I}$, with $F \cdot R=0$. Writing the $t$-linear part of $F$ as $\sum g_{i} t_{i}$, the $K S$ map sends $\partial / \partial t_{i}$ to the class of $g_{i}$ in $\operatorname{Hom}_{P / I}\left(I / I^{2}, P / I\right) \bmod$ derivations.

Suppose $\bar{W} \subset \mathbb{P}^{n+k}$ is another extension, with equations and relations given by $\bar{F}, \bar{g}^{I}, \bar{R}, \bar{u}^{I}$, and with the same $K S$ map. For each $i$ the classes of $g_{i}$ and $\bar{g}_{i}$ are the same, so there are derivations $D_{i}=\sum a_{i \alpha} \partial / \partial z_{\alpha}$ for which

$$
g_{i}-\bar{g}_{i}=D_{i} f+b_{i} \cdot f \text {, where } b_{i} \text { is a } \kappa \times \kappa \text { matrix. }
$$


(Note that if the generators of $I$ have the same degree, then each $b_{i}$ is 0 .) Replace $\bar{F}$ by $\bar{F}+\sum t_{i} b_{i} \cdot \bar{F}$, then apply the coordinate change $x_{\alpha}$ goes to $x_{\alpha}-\sum a_{i \alpha} t_{i}$; one obtains an extension equivalent to $\bar{W}$, with $g_{i}=\bar{g}_{i}$, all $i$.

Assume inductively that for all $I$ with $|I| \leq m, g^{I}=\bar{g}^{I}$ and for all $I$ with $|I|<m, u^{I}=\bar{u}^{I}$. Writing the coefficient of $g^{I}$ in the equations $F \cdot R=\bar{F} \cdot \bar{R}=0$, one deduces

$$
f \cdot u^{I}+(\cdots)=0 \text { and } f \cdot \bar{u}^{I}+(\cdots)=0,
$$

where the expressions in parentheses are the same. Therefore, $f \cdot\left(u^{I}-\bar{u}^{I}\right)=0$, so that

$$
u^{I}-\bar{u}^{I}=r \cdot b^{I}
$$

Thus, replacing $\bar{R}$ by $\bar{R}+\sum t^{I} \bar{R} \cdot b^{I}$ (sum over all $I$ with $|I|=m$ ) gives another relation vector, now with $u^{I}=\bar{u}^{I}$, all $|I| \leq m$. Next fix an $I$ with $|I|=m+1$. Again, one has equations

$$
f \cdot u^{I}+(\cdots)+g^{I} \cdot r=0 \text { and } f \cdot \bar{u}^{I}+(\cdots)+\bar{g}^{I} \cdot r=0,
$$

where the expressions in parentheses are equal to each other, by the inductive hypothesis. Thus,

$$
\left(g^{I}-\bar{g}^{I}\right) \cdot r=f \cdot v^{I}
$$

whence $g^{I}-\bar{g}^{I}$ defines a homomorphism of $I$ into $P / I$ of degree $-(m+1)$. But the hypothesis $\Gamma\left(N_{V / H}(-2)\right)=0$ implies also that $\Gamma\left(N_{V / H}(-i)\right)=0, i \geq 2$, whence (1.6.1) a homomorphism $I \rightarrow P / I$ of degree $\leq-2$ must be 0 . Thus, one can write

$$
g^{I}-\bar{g}^{I}=f \cdot w^{I} .
$$

Replacing $\bar{F}$ by $\bar{F}+\sum t^{I} \bar{F} \cdot w^{I}$ (over all $I$ with $|I|=m+1$ ), one may assume $g^{I}=\bar{g}^{I}$, all such $I$, so the induction may continue. In this way, since the sum is finite by degree considerations, one may conclude that $W$ and $\bar{W}$ are equivalent.

Next, fix a $k$-step extension $W$ with $K S$ an isomorphism; represent it as above by equations

$$
F=f+\sum t_{i} g_{i}+\sum t^{I} g^{I}
$$

Consider an arbitrary $j$-step extension $W^{\prime}$, with linear section defined by the vanishing of coordinates $s_{1}, s_{2}, \cdots, s_{j}$. Since the classes $\left[g_{i}\right]$ form a basis, one can after allowable linear coordinate changes in $\mathbb{P}^{n+j}$ write the equations for $W^{\prime}$ as

$$
\bar{F}=f+\sum^{\prime} s_{i} g_{i}+\sum s^{I} h^{I}
$$

where the first sum involves only the first $j^{\prime}$ variables (some $j^{\prime} \leq j$ ). Using $W$, one may write down another extension in $\mathbb{P}^{n+j}$ with the same $K S$ map; it is the cone over 
the subextension in $\mathbb{P}^{n+j^{\prime}}$

$$
F^{*}=f+\sum^{\prime} s_{i} g_{i}+\sum^{\prime} s^{I} g^{I}
$$

where both sums involve only the first $j^{\prime}$ variables. By (a), this extension is isomorphic to $W^{\prime}$.

\section{Remarks.}

(1.10.1). It is proved in ([W1], (2.8)) that the vanishing condition (1.9.1) is automatic when $V \subset \mathbb{P}^{n}$ is smooth and satisfies $\left(N_{2}\right)$ : that is, when $I(V)$ is generated by quadrics and the relations are generated by linear ones.

(1.10.2). Presumably one could prove a universality result for an extension $V \subset$ $W$ without assuming (1.9.1). One should require then that if $\Gamma\left(N_{V}(-i)\right)=0$, all $i>d$, then the Kodaira-Spencer maps $K S_{i}$ are surjective for all $i<d$, and an isomorphism for $i \geq d$. (Compare (1.5.2).) However, this would need to be worked out.

(1.10.3). For $n \geq 3$ the Segre embedding $\mathbb{P}^{1} \times \mathbb{P}^{n-1} \subset \mathbb{P}^{2 n-1}$ gives an extension of the twisted rational curve of degree $n$; it is universal for $n \neq 4$, but the KodairaSpencer map is not surjective once $n>3$.

(1.10.4). This Theorem allows a somewhat simpler proof of $S$. Mukai's classification [M2] of Gorenstein Fano 3-folds $X$, with $-K$ a generator of $\operatorname{Pic}(S)$, and with $g=7,8$, or 9: they are linear sections of an appropriate homogeneous space. Mukai shows that for the anticanonical embedding of $X$, the general linear section of codimension 2 is a canonical curve $C$, which (by his earlier work) is a linear section of a certain $G / P \subset \mathbb{P}^{N}$. One claims that $X$ is also a linear section of $G / P$, as will follow from 1.9 applied to the extension $C \subset G / P$. That the canonical embedding of $C$ satifies (1.9.1) follows, e.g., from (4.1.2) below plus results of [M1]. $G / P$ is smooth, hence not a cone, so the $K S$ map of this extension of $C$ is injective (by 1.9). But $K S$ is in fact bijective, by a dimension count; $\operatorname{dim}(G / P)-1$ is the corank of the Gaussian map - cf. [CM], ([W2], (6.5)).

\section{2. $K S$ AND THE NORMAL BUNDLE SEQUENCE}

(2.1) We frequently compose the Kodaira-Spencer map with a coboundary, because of the simple

Lemma 2.2. Let $V \subset H=\mathbb{P}^{n}$ be a smooth linearly normal subvariety, and $V \neq \mathbb{P}^{1}$. Then the coboundary map associated to the normal bundle sequence

$$
\delta: \Gamma\left(V, N_{V / H}(-1)\right) / \Gamma\left(H, \Theta_{H}(-1)\right) \rightarrow H^{1}\left(\Theta_{V}(-1)\right)
$$

is injective. 
Proof. By the usual long exact sequence in cohomology of the normal bundle sequence for $V \subset H$, it suffices to show that

$$
\Gamma\left(\Theta_{H}(-1)\right) \cong \Gamma\left(\Theta_{H}(-1) \otimes \mathcal{O}_{V}\right) .
$$

By the standard presentation of $\Theta_{H}$, this isomorphism is equivalent to the injectivity of a map

$$
H^{1}\left(\mathcal{O}_{V}(-1)\right) \rightarrow H^{1}\left(\mathcal{O}_{V}^{n+1}\right) .
$$

When $\operatorname{dim}(V) \geq 2$, the first group is 0 by Kodaira vanishing. When $\operatorname{dim}(V)=1$, by duality (and linear normality) one needs the surjectivity on the curve $V$ of

$$
\Gamma\left(K_{V}\right) \otimes \Gamma(\mathcal{O}(1)) \rightarrow \Gamma\left(K_{V}(1)\right),
$$

which is a well-known result of Petri when $V \neq \mathbb{P}^{1}$.

\section{Remarks.}

(2.3.1). Similarly, one can show that the coboundary $\delta$ is an isomorphism when $H^{1}\left(\mathcal{O}_{V}\right)=0$ and either $\operatorname{dim}(V) \geq 3$, or $\operatorname{dim}(V)=2$ and $\Gamma\left(K_{V}\right) \otimes \Gamma(\mathcal{O}(1)) \rightarrow$ $\Gamma\left(K_{V}(1)\right)$ is surjective.

(2.3.2). We conclude that the Kodaira-Spencer map for an extension of a linearly normal subvariety $V \subset \mathbb{P}^{n}$ has rank at most $h^{1}\left(\Theta_{V}(-1)\right)$.

(2.4) We can now relate the Kodaira-Spencer map of an extension to the class of the normal bundle sequence of $V \subset W$. Assume $V \subset H=\mathbb{P}$ is smooth and projectively normal, and $\left(V, H, W, \mathbb{P}^{n+k}\right)$ is a k-step extension.

(2.5) The normal bundle of $V$ in $W$ is the restriction of the normal bundle of $H \subset \mathbb{P}$; tensoring the surjection

$$
\Gamma\left(\mathcal{I}_{H}(1)\right) \otimes \mathcal{O}_{\mathbb{P}}(-1) \rightarrow \mathcal{I}_{H}
$$

with $\mathcal{O}_{H}$, dualizing, and restricting, one has

$$
N_{V / W} \cong \Gamma\left(\mathcal{I}_{H}(1)\right)^{*} \otimes \mathcal{O}_{V}(1) .
$$

So, one has an identification

$$
\Gamma\left(N_{V / W}(-1)\right) \cong \Gamma\left(\mathcal{I}_{H}(1)\right)^{*} .
$$

The normal bundle sequence of $V$ in $W$

$$
0 \rightarrow \Theta_{V} \rightarrow \Theta_{W} \otimes \mathcal{O}_{V} \rightarrow N_{V / W} \rightarrow 0
$$

is therefore determined as a bundle extension by a map

$$
\gamma: \Gamma\left(\mathcal{I}_{H}(1)\right)^{*} \rightarrow H^{1}\left(\Theta_{V}(-1)\right) .
$$

Proposition 2.6. Let $V \subset \mathbb{P}^{n}=H$ be smooth and projectively normal $\left(V \neq \mathbb{P}^{1}\right)$, $W \subset \mathbb{P}^{n+k}=\mathbb{P}$ a k-step extension. Then the bundle extension map $\gamma$ above is the negative of the composed map $\delta \cdot K S$. 
Proof. Choose coordinates as before, with $I \subset P$, and let $\bar{I}=I+\left(t_{1}, \cdots, t_{k}\right) \subset \bar{P}=$ $P\left[t_{i}\right]$. From the exact sequence

$$
0 \rightarrow N_{V / H} \rightarrow N_{V / \mathbb{P}} \rightarrow N_{H / \mathbb{P}} \otimes \mathcal{O}_{V} \rightarrow 0
$$

and [1.6.1), one deduces that $\Gamma\left(N_{V / \mathbb{P}}(-1)\right)$ consists of degree -1 homomorphisms $\bar{I} / \bar{I}^{2} \rightarrow \bar{P} / \bar{I}=P / I$. The subspace $\Gamma\left(N_{V / H}(-1)\right)$ consists of homomorphisms for which the $t_{i}$ go to 0 .

For an extension $W \subset \mathbb{P}$, each $f \in I$ lifts to $F \in I(W) \subset \bar{I}$, where $F=$ $f+\sum t_{i} g_{i}+\cdots$. Thus, $K S\left(\partial / \partial t_{\mu}\right)$ is the class of the homomorphism in $\Gamma\left(N_{V / \mathbb{P}}(-1)\right)$ sending $f$ to $g_{\mu}$, and every $t_{i}$ to 0 .

The map $\gamma$ factors through $\Gamma\left(\mathcal{I}_{H}(1)\right)^{*} \cong \Gamma\left(N_{V / W}(-1)\right) \subset \Gamma\left(N_{V / \mathbb{P}}(-1)\right)$ (an extension provides a splitting of 2.6.1). Now, $\partial / \partial t_{\mu}$ sends $t_{\nu}$ to $\delta_{\nu \mu}$; since

$$
f=-\sum t_{i} g_{i}-\cdots
$$

on $W, \partial / \partial t_{\mu}$ sends $f$ to $-g_{\mu}$.

Thus $(\gamma+\delta \cdot K S)\left(\partial / \partial t_{\mu}\right)$ is represented in $\Gamma\left(N_{V / \mathbb{P}}(-1)\right)$ by a map sending each $f \in I$ to 0 and $t_{i}$ to $\delta_{i \mu}$. This is exactly the image of the element " $\partial / \partial t_{\mu}$ " via

$$
\Gamma\left(\Theta_{\mathbb{P}}(-1)\right) \rightarrow \Gamma\left(\Theta_{\mathbb{P}} \otimes \mathcal{O}_{V}(-1)\right) \rightarrow \Gamma\left(N_{V / \mathbb{P}}(-1)\right) .
$$

Since the cokernel of the last map is contained in $H^{1}\left(\Theta_{V}(-1)\right)$, we have the assertion of the Proposition.

Remark 2.7. Let us denote the composed Kodaira-Spencer map $\delta \cdot K S$ by $\overline{K S}$; this map has target space $H^{1}\left(\Theta_{V}(-1)\right)$. We shall also abuse notation and not distinguish between $\overline{K S}$ and its negative $\gamma$.

Theorem 2.8. Suppose $V \subset \mathbb{P}^{n}$ is a projectively normal embedding of a smooth variety $\left(V \neq \mathbb{P}^{1}\right)$, for which $\Gamma\left(N_{V / \mathbb{P}^{n}}(-2)\right)=0$. Let $W \subset \mathbb{P}^{n+k}$ be an extension. From the normal sequence of $V$ in $W$ consider the coboundary map

$$
\gamma: \Gamma\left(N_{V / W}(-1)\right) \rightarrow H^{1}\left(\Theta_{V}(-1)\right) .
$$

Assume either

(i) $\operatorname{dim} V=1$ and $\operatorname{crk} \gamma=\operatorname{crk} \Phi\left(K_{V}, \mathcal{O}_{V}(1)\right)$ ( $\Phi$ a Gaussian)

(ii) $\operatorname{dim} V \geq 2$ and $\gamma$ is an isomorphism.

Then $W$ is a universal extension of $V$.

Proof. By Theorem 1.9, one must show that $K S$ is an isomorphism; composing with the injective coboundary map $\delta$, via (2.6) it suffices to consider $\gamma$. It remains necessary only to add that for a projectively normal curve, the coboundary map $\delta$ has the same corank as the Gaussian $\Phi\left(K_{V}, \mathcal{O}_{V}(1)\right)$, by e.g. [CM], (1.2). 


\section{A Du Val-type construction of extensions}

(3.1) For $E \subset X$ a smooth Cartier divisor on a projective variety, the normal class is the element in $H^{1}\left(\Theta_{E}(-E)\right)$ given by extension class of the normal bundle sequence

$$
0 \rightarrow \Theta_{E} \rightarrow \Theta_{X} \otimes \mathcal{O}_{E} \rightarrow \mathcal{O}_{E}(E) \rightarrow 0 .
$$

Let $Z \subset E$ be an effective Cartier divisor, $\pi: \tilde{X}=B l_{Z} X \rightarrow X$ be the blow-up of the ideal sheaf $\mathcal{I}_{Z}$ of $Z$ in $X, \tilde{Z} \subset \tilde{X}$ the exceptional divisor, and $\tilde{E} \subset \tilde{X}$ the proper transform of $E$. Via the isomorphism

$$
\left.\pi\right|_{\tilde{E}}: \tilde{E} \cong E
$$

one can identify the normal bundles

$$
N_{\tilde{E} / \tilde{X}} \cong N_{E / X}(-Z) \text {. }
$$

In particular, the normal sequences are comparable:

$$
\begin{gathered}
0 \rightarrow \Theta_{\tilde{E}} \rightarrow \Theta_{\tilde{X}} \otimes \mathcal{O}_{\tilde{E}} \rightarrow \mathcal{O}_{E}(E-Z) \rightarrow 0 \\
\| \quad \cap \quad \cap \quad \cap \\
0 \rightarrow \Theta_{E} \rightarrow \Theta_{X} \otimes \mathcal{O}_{E} \rightarrow \mathcal{O}_{E}(E) \rightarrow 0 .
\end{gathered}
$$

Thus, the normal classes of $E \subset X$ and $\tilde{E} \subset \tilde{X}$ are related via

$$
H^{1}\left(\Theta_{E}(-E)\right) \rightarrow H^{1}\left(\Theta_{E}(-(E-Z))\right)=H^{1}\left(\Theta_{\tilde{E}}(-\tilde{E})\right) .
$$

(3.2) For a line bundle $L$ on $X$ one has an exact sequence

$$
0 \rightarrow L(-E) \rightarrow \mathcal{I}_{Z} \cdot L \rightarrow L \otimes \mathcal{O}_{E}(-Z) \rightarrow 0 .
$$

If the linear system associated to $W \equiv \Gamma\left(\mathcal{I}_{Z} L\right) \subset \Gamma(L)$ has base scheme exactly $Z$, then the rational map $X--\rightarrow \mathbb{P}\left(W^{*}\right)$ becomes a morphism

$$
\rho: \tilde{X} \rightarrow \mathbb{P}\left(W^{*}\right)
$$

associated to the line bundle $\pi^{*}(L)(-\tilde{Z})$. The linear system defining $\left.\rho\right|_{\tilde{E}}$ is given by the image of $W$ in $\Gamma\left(E, L \otimes \mathcal{O}_{E}(-Z)\right)$.

(3.3) Assuming $L=\mathcal{O}(E), \mathcal{O}_{E}(E-Z)$ is very ample, and $H^{1}\left(\mathcal{O}_{X}\right)=0$, one has the exact sequence

$$
0 \rightarrow \Gamma\left(\mathcal{O}_{X}\right) \rightarrow \Gamma\left(\mathcal{I}_{Z} \mathcal{O}(E)\right) \rightarrow \Gamma\left(E, \mathcal{O}_{E}(E-Z)\right) \rightarrow 0 .
$$

It follows that $Z$ is the base scheme associated to $\Gamma\left(\mathcal{I}_{Z} L\right)$. Note $H^{1}\left(\mathcal{O}_{\tilde{X}}\right)=0$ and $\mathcal{O}_{\tilde{E}}(\tilde{E}) \cong \mathcal{O}_{E}(E-Z)$ is very ample.

Lemma 3.4. Let $A$ be a smooth divisor on a projective variety $B$ with $H^{1}\left(\mathcal{O}_{B}\right)=0$. Suppose $\mathcal{O}_{A}(A)$ is very ample. Then $\mathcal{O}(A)$ is basepoint-free and gives a birational morphism $\rho: B \rightarrow \mathbb{P}\left(\Gamma(B, \mathcal{O}(A))^{*}\right)$. $\rho$ is an isomorphism in a neighborhood of $A$, and maps $A$ isomorphically to a hyperplane section $\bar{A}$ of $\rho(B) \equiv \bar{B}$. 
Proof. The morphism associated to $\mathcal{O}(A)$ clearly separates points of $A$ from points off $A$ and, as $\Gamma(\mathcal{O}(A)) \rightarrow \Gamma\left(\mathcal{O}_{A}(A)\right)$, from other points on $A$. By hypothesis tangent vectors on $A$ are separated; since $A$ is smooth, tangent vectors of $X$ at points of $A$ are separated.

Proposition 3.5. Let $E \subset X$ be a smooth Cartier divisor on a projective variety with $h^{1}\left(\mathcal{O}_{X}\right)=0, Z \subset E$ a divisor with $\mathcal{O}_{E}(E-Z)$ very ample. Then

(i) the linear system $\Gamma\left(\mathcal{I}_{Z} \mathcal{O}(E)\right)$ gives a (1-step) extension $\bar{X}$ of $\left(E, \mathcal{O}_{E}(E-Z)\right)$

(ii) the normal class of $E \subset \bar{X}$ is the image of the normal class of $E \subset X$ via $H^{1}\left(E, \Theta_{E}(-E)\right) \rightarrow H^{1}\left(E, \Theta_{E}(-(E-Z))\right)$, or arising from

$$
0 \rightarrow \Theta_{E}(-(E-Z)) \rightarrow \Theta_{X} \otimes \mathcal{O}_{E}(-(E-Z)) \rightarrow \mathcal{O}_{E}\left(\mathcal{O}_{E}(Z) \rightarrow 0\right.
$$

\section{Remarks.}

(3.6.1). In case $X=\mathbb{P}^{2}, E$ is a smooth cubic, and $Z \subset E$ is a scheme of length $\leq 6$, the construction above gives a Del Pezzo surface, viewed as an extension of $E$.

(3.6.2). The sequence [3.3.1] shows that the domain of the $K S$ map for the extension in (3.5.ii) is isomorphic to $\Gamma\left(\mathcal{O}_{X}\right)^{*}$, hence has a natural basis element. Thus, in case $\mathcal{O}_{E}(E-Z)$ is projectively normal, the $\overline{K S}$ map is described by the (negative of the) normal class of $E \subset \bar{X}$.

Corollary 3.7. Let $E$ and $F$ be effective Cartier divisors on a projective variety $X$, with $E$ smooth and $h^{1}\left(\mathcal{O}_{X}\right)=0$. Suppose $\mathcal{O}_{X}(E-F)$ is very ample. Then denoting $Z=E \cap F$ :

(3.7.1). The linear system of $\Gamma\left(\mathcal{I}_{Z} \mathcal{O}(E)\right)$ has base scheme $Z$ and defines an isomorphism off $\operatorname{Supp}(F)$.

(3.7.2). $\rho: \tilde{X}=B l_{Z} X \rightarrow \mathbb{P}\left(\Gamma\left(\mathcal{I}_{Z} \mathcal{O}(E)\right)^{*}\right)$ is an isomorphism off $\tilde{F}$, the proper transform of $F$, and collapses $\tilde{F}$ to a 0-dimensional scheme.

(3.7.3). $E \subset \rho(\tilde{X})=\bar{X} \subset \mathbb{P}\left(\Gamma\left(\mathcal{I}_{Z} \mathcal{O}(E)\right)^{*}\right)$ is an extension of $E$ embedded by the very ample line bundle $\mathcal{O}_{E}(E-F)$.

(3.7.4). The $\overline{K S} \operatorname{map}(2.7)$ for the extension $E \subset \rho(\tilde{X})$ is given by the composition

$$
\Gamma\left(\mathcal{O}_{E}\right) \rightarrow \Gamma\left(\mathcal{O}_{E}(F)\right) \rightarrow H^{1}\left(E, \Theta_{E}(-(E-F))\right) .
$$

Proof. Since the linear system of $\Gamma\left(\mathcal{I}_{Z} \mathcal{O}(E)\right)$ is generated by $E$ and $F+D$, where $D$ runs through the very ample system $|E-F|$, (3.7.1) follows.

For (3.7.2), let $U=\operatorname{Spec} R \subset X$ be an affine open neighborhood, with $E, F$ defined locally by $f, g \in R$, respectively. On $U$, the rational map may be written as

$$
\left[f, g, g h_{1}, \cdots, g h_{s}\right]
$$

where $\left(h_{1}, \cdots, h_{s}\right): U \rightarrow \mathbb{C}^{s}$ is an embedding. The complement of $\tilde{F}$ in $\pi^{-1}(U)$ is an affine $\tilde{U} \subset \tilde{X}$, with coordinate $\operatorname{ring} R[t] / f-t g$, with $\tilde{E}$ defined by $t, \tilde{Z}$ by $g$. On $\tilde{U}$, 
the rational map becomes a morphism

$$
\left[t, 1, h_{1}, \cdots, h_{s}\right]
$$

as this gives an embedding on $\mathbb{C} \times U$, which contains $\tilde{U}$, the first assertion follows. Note also that $\mathcal{I}_{Z} \mathcal{O}(E)$ restricted to $F$ is $\cong \mathcal{O}_{F}$. The remaining claims follow from Proposition 3.5.

(3.8) One may form $k$-step extensions in the DuVal-type construction above by considering the complete linear system of $Z$ on $E$. Let $Y$ be a projective variety with $h^{1}\left(\mathcal{O}_{Y}\right)=0, D \subset Y$ a smooth Cartier divisor, $M$ a line bundle on $D$ so that $\mathcal{O}_{D}(D-M)$ is very ample, and $V \subset \Gamma(D, M)$ a non-0 linear subspace. The linear system associated to $V$ is represented by $Z \subset D \times \mathbb{P}(V)$, a relatively effective Cartier divisor over $\mathbb{P}(V)$. In the previous notation, let

$$
\begin{aligned}
X & =Y \times \mathbb{P}(V) \\
E & =D \times \mathbb{P}(V) \\
Z & \subset D \times \mathbb{P}(V) \text { as above } \\
L & =\mathcal{O}_{Y}(D) \otimes \mathcal{O}(1) .
\end{aligned}
$$

Note that $\mathcal{O}_{E}(Z) \cong \mathcal{O}_{D}(M) \otimes \mathcal{O}(1)$. The restriction of $\mathcal{I}_{Z} L$ to $E$ is

$$
L \otimes \mathcal{O}_{E}(-Z) \cong \pi_{1}^{*} \mathcal{O}_{D}(D-M) .
$$

Since $H^{1}(X, L(-E)) \cong H^{1}\left(Y, \mathcal{O}_{Y}\right) \otimes H^{0}(\mathbb{P}(V), \mathcal{O}(1))=0$, one has the exact sequence

$$
0 \rightarrow \Gamma\left(\pi_{2}^{*} \mathcal{O}(1)\right) \rightarrow \Gamma\left(\mathcal{I}_{Z} L\right) \rightarrow \Gamma\left(\pi_{1}^{*} \mathcal{O}_{D}(D-M)\right) \rightarrow 0 .
$$

The first subspace is canonically $V^{*}$, and yields divisors of the form $E+\pi_{2}^{-1} H$, where $H \subset \mathbb{P}(V)$ is a hyperplane; so $\Gamma\left(\mathcal{I}_{Z} L\right)$ has no base points off $E$. $\mathcal{O}_{D}(D-M)$ is very ample, hence free, so there are no base points on $E$ except along $Z$. The map $\rho: \tilde{X} \rightarrow \mathbb{P}\left(\Gamma\left(\mathcal{I}_{Z} L\right)^{*}\right)=\mathbb{P}^{N}$ restricted to $\tilde{E} \cong E=D \times \mathbb{P}(V)$ is the projection map to $D$ followed by the embedding given by $\mathcal{O}_{D}(D-M)$. Intersecting the linear space $\mathbb{P}(V) \subset \mathbb{P}^{N}$ with $\rho(\tilde{X})$ and pulling back to $\tilde{X}$ is (as a scheme) the intersection of the divisors $E+\pi_{2}^{-1} H$, which equals $E$. We conclude that the smooth variety $\rho(\tilde{E})$ equals $\rho(\tilde{X}) \cap \mathbb{P}(V)$. In particular, $\rho$ is birational onto its image, which is a $(\operatorname{dim} V)$-step extension of $\rho(\tilde{E}) \cong D$, embedded via $\mathcal{O}_{D}(D-M)$. Note finally that $V$ is naturally isomorphic to $\Gamma\left(\mathcal{I}_{\mathbb{P}(V)}(1)\right)^{*}$. We change notation and summarize in the

Theorem 3.9. Let $E \subset X$ be a smooth Cartier divisor on a projective variety with $h^{1}\left(\mathcal{O}_{X}\right)=0, M$ a line bundle on $E$ with $\mathcal{O}_{E}(E-M)$ very ample.

(a) Then for every non-0 $V \subset \Gamma(E, M)$, there is a (dim $V)$-step extension $\bar{X}$ of $E$ embedded by $\mathcal{O}_{E}(E-M)$. 
(b) Assume further $\mathcal{O}_{E}(E-M)$ is projectively normal. Then the $\overline{K S}$ map of the extension $\bar{X}$ is the composition

$$
V \subset \Gamma(E, M) \rightarrow H^{1}\left(E, \Theta_{E}(-E) \otimes \mathcal{O}(M)\right),
$$

the second map arising from the normal sequence of $E \subset X$.

Corollary 3.10. Let $E \subset X$ be a smooth Cartier divisor on a normal projective variety with $h^{1}\left(\mathcal{O}_{X}\right)=0$. Assume $M$ a line bundle on $X$ so that

(a) $\mathcal{O}_{X}(E) \otimes M^{-1}$ is very ample

(b) $\mathcal{O}_{X}(E) \otimes M^{-1} \otimes \mathcal{O}_{E}$ is projectively normal.

Then there is a $h^{0}(M)$-step extension of $\left(E, \mathcal{O}_{E}(E) \otimes M^{-1}\right)$, whose 1-step sub-extensions are formed by blowing up $X$ at $E \cap F$ (where $F$ is effective, $\mathcal{O}(F) \cong M$ ), and blowing down each component of $\tilde{F}$.

If further

$$
\left.H^{0}\left(\Theta_{X} \otimes \mathcal{O}_{E}(-E) \otimes M\right)\right)=0
$$

then the Kodaira-Spencer map of the extension is injective.

Proof. By the Kodaira vanishing theorem for a normal variety, $h^{i}\left(X, \mathcal{O}_{E}(-E) \otimes M\right)=$ $0, i=0,1$, so $\Gamma(X, M) \cong \Gamma\left(E, M \otimes \mathcal{O}_{E}\right)$. The normal sequence of $E$ in $X$ gives the exact

$$
H^{0}\left(E, \Theta_{X} \otimes \mathcal{O}_{E}(-E) \otimes M\right) \rightarrow H^{0}\left(E, M \otimes \mathcal{O}_{E}\right) \rightarrow H^{1}\left(E, \Theta_{E}(-E) \otimes M\right) .
$$

Now all assertions follow from the Theorem.

\section{Remarks.}

(3.11.1). An extension constructed as in Corollary 3.10 has a non-rational singularity if $h^{n-1}\left(\mathcal{O}_{F}\right) \neq 0(n=\operatorname{dim} X)$. For, one blows up $X$ along $F \cap E$ and blows down $\tilde{F} \cong F$ via $\rho$, to a finite set of points. In the notation above, $R^{n-1} \rho_{*} \mathcal{O}_{\tilde{X}} \rightarrow$ $H^{n-1}\left(\mathcal{O}_{\tilde{F}}\right) \neq 0$.

(3.11.2). Suppose $\left(E, \mathcal{O}_{E}(E) \otimes M^{-1}\right)$ satisfies $\left(N_{2}\right)$ (or just (1.9.1)). A dimension count plus Corollary 3.10 can be used to show the constructed extension is universal. This is of special interest when $M=-K_{X}$.

Corollary 3.12. Let $E \subset X$ be a smooth Cartier divisor on a normal Gorenstein projective variety with $h^{1}\left(\mathcal{O}_{X}\right)=0$. Assume

(a) $K_{X}+E$ is very ample, and $K_{E}$ is projectively normal

(b) $h^{0}\left(E, \Theta_{X}\left(-\left(K_{X}+E\right)\right) \otimes \mathcal{O}_{E}\right)=0$

(c) For the embedding $E \subset \mathbb{P}=\mathbb{P}\left(\Gamma\left(K_{E}\right)^{*}\right)$ given via $K_{E}$,

$$
\begin{gathered}
\operatorname{dim} \Gamma\left(N_{E / \mathbb{P}}(-1)\right) / \Gamma\left(\Theta_{\mathbb{P}}(-1)\right)=h^{0}\left(-K_{X}\right) \\
\Gamma\left(N_{E / \mathbb{P}}(-2)\right)=0 .
\end{gathered}
$$


Then the construction in (3.8) gives a universal extension of $\left(E, K_{E}\right)$. Any nonconical 1-step extension of $\left(E, K_{E}\right)$ is constructed from an anti-canonical divisor $F$ on $X$, by blowing up $E \cap F$, and blowing down $\tilde{F}$. In particular, if $K_{X} \neq \mathcal{O}_{X}$, then every extension of $\left(E, K_{E}\right)$ has a non-rational singularity.

Proof. If $h^{0}\left(-K_{X}\right)=0$, by (c) the $K S$ map of any extension is 0 ; by Theorem 1.9, it must be a cone over $\left(E, K_{E}\right)$ (necessarily non-rational, as $K_{E}$ ample implies $\left.h^{n-1}\left(\mathcal{O}_{E}\right) \neq 0\right)$.

If $h^{0}\left(-K_{X}\right) \neq 0$, Corollary 3.10 applied to $M=-K_{X}$ gives an extension of $\left(E, K_{E}\right)$ with $K S$ an isomorphism (via (c)). By (1.9), it is universal, and every extension is obtained by blowing up $X$ along $E \cap F$, where $F \in\left|-K_{X}\right|$ is an anticanonical divisor. If $K_{X} \cong \mathcal{O}_{X}$, one obtains that $X$ is the essentially unique extension of $\left(E, K_{E}\right)$. If $K_{X} \cong \mathcal{O}_{X}(-F)$, with $F \neq 0$, then

$$
0 \rightarrow K_{X} \rightarrow \mathcal{O}_{X} \rightarrow \mathcal{O}_{F} \rightarrow 0
$$

and the equalities $h^{n}\left(\mathcal{O}_{X}\right)=h^{0}\left(K_{X}\right)=0, h^{n}\left(K_{X}\right)=1$ imply that $h^{n-1}\left(\mathcal{O}_{F}\right) \neq$ 0 . Applying Remark (3.11.1), one has a non-rational singularity on every 1-step extension.

Remark 3.13. It is important to note that the conditions of Corollary 3.12 hold for sufficiently ample $E$ on $X$; that is, for $D$ is ample and $n$ sufficiently large, a smooth $E$ in $|n D|$ satisfies (a)-(c). This is clear for (a) and (b), since $h^{1}\left(\mathcal{O}_{X}\right)=0$ implies the surjectivity of

$$
\Gamma\left(X, K_{X}+E\right) \rightarrow \Gamma\left(E, K_{E}\right) .
$$

For (c), it follows e.g. from [I] that for large $E, K_{X}+E$ satisfies the syzygy condition $\left(N_{2}\right)$. So it remains to check the dimension claim. If $\operatorname{dim} X \geq 3$, by $(2.3 .2)$ we need only show that for large E

$$
\operatorname{dim} H^{1}\left(\Theta_{E}\left(-K_{E}\right)\right)=h^{0}\left(-K_{X}\right) .
$$

The normal bundle sequence for $E \subset X$ makes clear that for $E$ large the first term is $h^{0}\left(E,-K_{E}\right)$, which in turn equals the second term for $E$ large. When $\operatorname{dim} X=2$, the result is well-known (e.g., [DM] or (4.4) below).

\section{THE CASE OF CURVES}

(4.1) If $C$ is a smooth non-hyperelliptic curve of genus $g \geq 3$, then $K_{C}$ is very ample, and the canonical embedding $C \subset \mathbb{P}^{g-1}=\mathbb{P}$ is projectively normal (Noether's theorem). An extension $W \subset \mathbb{P}^{g}$ of $C$ is a normal Gorenstein surface with $K_{W} \cong \mathcal{O}_{W}$ and $h^{1}\left(\mathcal{O}_{W}\right)=0$ (a "canonically trivial surface.") To apply Corollary 3.12, note

(4.1.1). $\operatorname{dim} H^{0}\left(N_{\mathbb{P}}(-1)\right) / H^{0}\left(\Theta_{\mathbb{P}}(-1)\right)=$ crk $\Phi_{K}: \Lambda^{2} \Gamma(K) \rightarrow \Gamma\left(K^{\otimes 3}\right)=$ corank of Gaussian-Wahl map [W1]. 
(4.1.2). $H^{0}\left(N_{C / \mathbb{P}}(-i)\right)=0, i \geq 2$, unless $C$ is trigonal of genus $\leq 10$; the intersection of a del Pezzo surface in $\mathbb{P}^{g-1}$ with a quadric $(g \leq 10)$; or is bielliptic.

(4.1.2) asserts the Gaussian $\Phi\left(K, K^{\otimes i}\right)$ is surjective for $i \geq 2$ except in these cases. When the Clifford index $\geq 3$, one has property $\left(N_{2}\right)$ (by $[\mathrm{S}],[\mathrm{V}]$ ); so recall $(1.10 .1)$. The other cases can be deduced via $[\mathrm{CM}] ;[\mathrm{T}]$, p. 161; and [W2], §5.8.

(4.2) Conditions (b) and (c) in Corollary 3.12 arise dually in the

Proposition 4.3. (cf. $[D M])$. Let $C \subset X$ be a smooth Cartier divisor on a normal Gorenstein surface with $h^{1}\left(\mathcal{O}_{X}\right)=0$. Assume

(a) $H^{1}\left(\Omega_{X}^{1}\left(2 K_{X}+2 C\right) \otimes \mathcal{O}_{C}\right)=0$

(b) $K_{X}+C$ is ample

(c) The Gaussian $\Phi_{K_{X}+C}$ is surjective

(d) $\quad H^{1}\left(\Omega_{X}^{1}\left(2 K_{X}+C\right)\right)=0$.

Then

$$
\operatorname{corank} \Phi_{K}=h^{0}\left(-K_{X}\right)
$$

i.e., "X computes the Gaussian of $C$."

Proof. A straightforward diagram chase as in [DM], Lemma 2.6, except that one uses $h^{i}\left(X, \mathcal{O}_{X}\left(-\left(K_{X}+C\right)\right)\right)=0(i=0,1)$, by Kodaira Vanishing for a normal surface.

(4.4) Of course sufficiently ample $C$ on $X$ satisfy conditions (a)-(d) of the Proposition. We paraphrase Corollary 3.12 as follows:

Theorem 4.5. Suppose $X$ is a Gorenstein projective surface with $h^{1}\left(\mathcal{O}_{X}\right)=0$. Let $C \subset X$ be a smooth Cartier divisor, not one of the special curves of genus $\leq 10$ of (4.1.2, nor bielliptic. Assume

(i) $K_{X}+C$ is very ample

(ii) $X$ computes the Gaussian of $C$.

Then every extension of the canonical embedding of $C$ is constructed as in $§ 3$ from an anti-canonical divisor on $X$. In particular, $C$ does not sit on a $K-3$ surface, unless $X$ is a $K-3$ surface.

Remark 4.6. In the moduli space $\mathcal{M}_{g}$, there are generally many irreducible components of the locus of curves with Gaussian corank 1, besides the $(g+19)$-dimensional stratum of $K-3$ curves. For instance, let $X \subset \mathbb{P}^{g^{\prime}}$ be the cone over a general canonical curve $C^{\prime}$ of genus $g^{\prime} \geq 12$. By the Theorem, a smooth hypersurface section $C$ of degree $d$ large has genus $g=d^{2}\left(g^{\prime}-1\right)+1$ and Gaussian corank 1, and uniquely determines $X$ (hence $C$ ). Varying $C^{\prime}$ and the hypersurface section gives a $\left(g+2 g^{\prime}-3\right)$-dimensional locus in $\mathcal{M}_{g}$, with Gaussian corank 1. 
(4.7) One can say precisely which complete intersection curves sit on $K-3$ surfaces, and more generally describe the canonically trivial surfaces of which they are hyperplane sections. Let $C \subset \mathbb{P}^{n}$ be a complete intersection curve of multidegree $2 \leq d_{1} \leq d_{2} \cdots \leq d_{n-1} \equiv d$; assume $g \geq 2$, so $\kappa=\sum d_{i}-(n+1)>0$ and $K_{C}=\mathcal{O}_{C}(\kappa)$. Let $X$ be a general complete intersection surface of multidegree $d_{1} \leq d_{2} \leq \cdots \leq d_{n-2}$ which contains $C$ ( $X$ is unique unless $\left.d_{n-2}=d_{n-1}\right)$; then $K_{X}=\mathcal{O}_{X}(\kappa-d)$. By Theorem 6.2 of [W1], $\kappa>d$ implies the Gaussian of $C$ is surjective, so the canonical embedding of $C$ has only conical extensions.

Lemma 4.8. $X$ computes the Gaussian of $C$, except in the following cases:

$$
\begin{array}{ll}
n=2, & d_{1} \leq 6 \\
n=3, & d_{1} \leq d_{2} \leq 4 \\
n=4, & \left(d_{1}, d_{2}, d_{3}\right)=(2,2,2),(2,2,3),(2,3,3) \\
n=5, & \text { all } d_{i}=2 .
\end{array}
$$

Proof. $\mathcal{O}_{X}(C)=\mathcal{O}_{X}(d)$, and $K_{X}+C=\mathcal{O}_{X}(\kappa)$ is very ample. By (4.3), it suffices to check that except in the cases above, the Gaussian of $\mathcal{O}_{X}(\kappa)$ is surjective and

$$
H^{1}\left(\Omega_{X}^{1}(2 \kappa) \otimes \mathcal{O}_{C}\right)=H^{1}\left(\Omega_{X}^{1}(2 \kappa-d)\right)=0
$$

As in [W1], 6.6, the first condition follows from the surjectivity of non-trivial Gaussians on $\mathbb{P}^{n}$ plus surjectivity of the composition

$$
\Gamma\left(\mathbb{P}^{n}, \Omega_{\mathbb{P}}^{1}(2 \kappa)\right) \rightarrow \Gamma\left(X, \Omega_{\mathbb{P}}^{1}(2 \kappa) \otimes \mathcal{O}_{X}\right) \rightarrow \Gamma\left(X, \Omega_{X}^{1}(2 \kappa)\right)
$$

Remark 4.9. If $n \geq 2$, in all above examples $X$ is a canonically trivial surface (a $K-3$, if smooth), but it is not unique; thus $C$ could not have Gaussian of corank $1=$ $h^{0}\left(-K_{X}\right)$.

Theorem 4.10. Let $C \subset \mathbb{P}^{n}$ be a smooth complete intersection curve. Aside from (4.8.1] (4.8.4), the only $C$ which could lie on a K-3 surface are the "obvious ones," with multi-indices

(4.10.1). $\quad n=3,\left(4, d_{2}\right), d_{2}>4$

(4.10.2). $\quad n=4,\left(2,3, d_{3}\right), d_{3}>3$

(4.10.3). $\quad n=5,\left(2,2,2, d_{4}\right), d_{4}>2$.

In these cases, the surface $X$ is a $K-3$ (if smooth) and is a universal extension of the canonical curve.

Proof. (4.10.1)-(4.10.3) are exactly the cases with $\kappa=d>d_{n-2}$; the other $\kappa=d$ examples are covered by (4.8). So Theorem 4.5 applied to $X$ gives the result, once we show the special curves of (4.1.2) are included in (4.8). For instance, it suffices to check that the Gaussians $\Phi\left(K, K^{\otimes i}\right)$ on $C$ are surjective, all $i \geq 2$. Restricting from $\mathbb{P}^{n}$ as in (4.8), one needs that the composition

$$
\Gamma\left(\mathbb{P}^{n}, \Omega_{\mathbb{P}^{n}}^{1}((i+1) \kappa)\right) \rightarrow \Gamma\left(C, \Omega_{\mathbb{P}^{n}}^{1}((i+1) \kappa) \otimes \mathcal{O}_{C}\right) \rightarrow \Gamma\left(C, \Omega_{C}^{1}((i+1) \kappa)\right)
$$


is surjective for $i \geq 2$; a standard computation gives this once $2 \kappa>d$. But the cases with $2 \kappa \leq d$ are included in (4.8).

\section{Remarks.}

(4.11.1). Taking double covers, smooth plane curves of degree 4, 5, or 6 lie on a $K-3$ (possibly with rational double points) - cf. the proof of (5.5) below.

(4.11.2). Green-Lazarsfeld [GL] already had shown that a plane curve of degree $d \geq 7$ does not lie on a $K-3$; they argue that the $g_{d}^{2}$ would be induced by a divisor on the $K-3$, from which a contradiction follows.

Proposition 4.12. Let $C$ be a smooth curve on the rational ruled surface $\mathbb{F}_{n}$, linearly equivalent to $p B+q F$ (where $B$ is the section with $B \cdot B=-n, F$ is a fibre). Assume that $p \geq 5$, and

(a) if $n=0$, then $q \geq 5$

(b) if $n=1$, then $q \geq p+4$

(c) if $n=2$, then $q \geq 2 p+2$.

Then $\mathbb{F}_{n}$ computes the Gaussian of $C$, with $h^{0}\left(\mathbb{F}_{n}, \mathcal{O}(-K)\right)=n+6$ if $n \geq 3$ (and $=9$ for $n \leq 3)$. Every extension of the canonical embedding of $C$ arises from blowing up $\mathbb{F}_{n}$ and blowing down an anti-canonical divisor. In particular, $C$ does not sit on a K-3 surface.

Proof. The first assertion is Theorem 5.5 of [DM]. Since $K=-2 B-(n+2) F$, and $r B+s F$ is very ample iff $r \geq 1$ and $s>r n$, we conclude that $K+C$ is very ample. Theorem 4.5 applies once we note $C$ can be neither trigonal, nor on a del Pezzo, nor bielliptic, as seen by comparing the genera and corank of the Gaussian of such curves from $[\mathrm{Br}],[\mathrm{CM}]$ with the formulas above when $C \subset \mathbb{F}_{n}$.

\section{Remarks.}

(4.13.1). Blowing up $\mathbb{P}^{2}$ at a point of a smooth curve $C$, the proper transform on $\mathbb{F}_{1}$ is very ample but with Gaussian of corank 10, not 9 - thus the restrictions above for small $n$.

(4.13.2). When $n \geq 3,-K$ has $B$ as base curve. One sees that a generic extension of $C \subset \mathbb{P}^{g-1}$ has a non-smoothable cusp singularity with two exceptional curves in the minimal resolution.

(4.14) Now suppose $C$ is bielliptic, of genus $\geq 6$. Then $C \subset \mathbb{P}^{g-1}$ is the complete intersection of a quadric $Q$ with $X$, a projective cone over an elliptic curve $D$ of degree $g-1$ in $\mathbb{P}^{g-2}[\mathrm{CM}]$. It is known that

$$
\begin{aligned}
& \operatorname{dim} H^{0}\left(C, N_{C / \mathbb{P}}(-1)\right) / \Gamma\left(\Theta_{\mathbb{P}}(-1)\right)=2 g-2 \\
& \operatorname{dim} H^{0}\left(C, N_{C / \mathbb{P}}(-i)\right) \quad=1 \quad i=2 \\
& =0 \quad i \geq 3 \text {. }
\end{aligned}
$$


$D \subset \mathbb{P}^{g-2}$ satisfies $\left(N_{2}\right)$; it is defined by quadratic equations $f_{1}, \cdots, f_{N}$, in variables $x_{2}, \cdots, x_{g}$. We may assume coordinates chosen in $\mathbb{P}^{g-1}$ so that $C$ is defined by these equations plus another of the form $h=x_{1}^{2}-A\left(x_{2}, \cdots, x_{g}\right)$, where $A$ is also quadratic.

Theorem 4.15. (cf. [Re]). Suppose $C$ is bielliptic of genus $g \geq 11$. Then there is a unique nontrivial extension of the canonical embedding of $C$, a birationally ruled surface with two simple elliptic singularities of degree $(g-1)$. In particular, $C$ does not sit on a K-3 surface.

Proof. $C$ is defined by $f_{1}, \cdots, f_{N}$, and $h$, and the relations are generated by the linear relations among the $f_{i}$, plus the trivial relations $f_{i} \cdot h-h \cdot f_{i}=0$ (since $h$ is not a 0-divisor mod the $f_{i}$ ). Let $W \subset \mathbb{P}^{g}$ be an extension of $C \subset \mathbb{P}^{g-1}$, defined by $t=0$. Then liftings of the equations $f_{i}$ induce an extension of $X$ as well. But it is well-known $([\mathrm{P}])$ that the cone over an elliptic curve of degree $\geq 10$ has no non-trivial extensions; thus, when $g-1 \geq 10$, after changing coordinates one may assume the equations defining $W$ are given by $f_{1}, \cdots f_{N}, h+t \alpha+t^{2} \beta$. Given the form of $h$, replacing $x_{1}$ by $x_{1}-t \alpha / 2$ changes only the last equation, to $h+t^{2} \beta$, where $\beta \in \mathbb{C}$. If $\beta=0$, one has a cone; otherwise, replacing $t$ by $t / \sqrt{\beta}$, one has the unique non-trivial extension of $C$ (with $\beta=1$ ). This is described geometrically via Corollary 3.7. Noting $K_{X} \cong \mathcal{O}_{X}(1)$, let $E=C$ and $F \cong D$ a smooth hyperplane section of $X \subset \mathbb{P}^{g-1}$. Blowing up the $2(g-1)$ points of $C \cap D$, then blowing down the proper transform of $D$, gives an extension of $C$ with a second elliptic singularity, also of degree $g-1$. Note that the $K S$ map is 0 for this extension.

\section{Complete intersections which lie on Calabi-Yaus}

(5.1) Let $X \subset \mathbb{P}^{n}$ be a smooth complete intersection subvariety of dimension $r \geq 2$, of multidegree $2 \leq d_{1} \leq \cdots \leq d_{n-r}$. Assume $\kappa=\sum d_{i}-(n+1)>0$, so that $X$ has very ample canonical bundle $\mathcal{O}_{X}(\kappa)$. Let $Y_{\alpha} \supset X$ be a general complete intersection defined by the same equations as $X$ except for one of degree $d_{\alpha}$. Then $K_{Y_{\alpha}} \cong \mathcal{O}_{Y_{\alpha}}\left(\kappa-d_{\alpha}\right)$. Thus, if $\kappa=d_{\alpha}$ for some $\alpha$, then $X$ is a hyperplane section of a Calabi-Yau, assuming $Y_{\alpha}$ can be chosen to have rational singularities. More generally,

Proposition 5.2. Let $X$ be a complete intersection so that $\kappa$ divides $d_{\alpha}$, for some $\alpha$. Suppose $Y=Y_{\alpha}$ can be chosen to have at most rational singularities. Then there is a cyclic branched cover of $Y$ which is a Calabi-Yau variety and on which $X$ is a hyperplane section.

Proof. If $m \kappa=d_{\alpha}$, then $\mathcal{O}_{Y}(X)=\mathcal{O}_{Y}(m \kappa)$; form the $m$-fold cyclic branched cover of $Y$ along $X$. It is easy to check $Y$ is a Calabi-Yau variety, and the reduced ramification divisor $X^{\prime} \cong X$ is very ample.

(5.3) If $\kappa$ is large compared to the $d_{\alpha}$, then $X$ cannot sit on a Calabi-Yau, as is proved for curves in (4.10). Specifically, 
Theorem 5.4. Let $X \subset \mathbb{P}^{n}$ be a smooth complete intersection, with multidegree $d_{1} \leq$ $\cdots \leq d_{n-r-1} \leq d_{n-r}=d$, and with $\kappa=\sum d_{i}-(n+1)>0$.

(a) If $\kappa>d$, then $\left(X, K_{X}\right)$ has no extensions.

(b) If $d>\kappa>\frac{d}{2}$ and $\kappa>d_{n-r-1}$, then $\left(X, K_{X}\right)$ has non-trivial extensions, but all have non-rational singularities.

Corollary 5.5. A smooth hypersurface $X \subset \mathbb{P}^{n}$ of degree $d$ is a hyperplane section of a Calabi-Yau variety iff $n+1 \leq d \leq 2 n+2$.

Corollary 5.6. Let $X \subset \mathbb{P}^{n}$ be a smooth complete intersection of type $\left(d^{\prime}, d\right)$, with $d^{\prime} \leq d$. Then

(a) if $d \leq n+1, X$ sits on a smooth Calabi-Yau hypersurface.

(b) if $d>n+2$ and $\frac{d}{2}+d^{\prime}>n+1$, then $X$ cannot sit on a Calabi-Yau variety unless $d^{\prime}=n+1$.

(c) if $\frac{d}{2}+d^{\prime}=n+1$, then the generic complete intersection variety of type $\left(d^{\prime}, d\right)$ sits on a Calabi-Yau.

Proof of 5.4. We assume $2 \kappa>d$. Standard cohomological arguments yield,

$$
\begin{aligned}
H^{1}\left(\Theta_{X}(-i \kappa)\right) & =0, i \geq 2, \\
H^{1}\left(\Theta_{X}(-\kappa)\right) & \cong \oplus \Gamma\left(\mathcal{O}_{X}\left(d_{i}-\kappa\right)\right) .
\end{aligned}
$$

It follows from the normal bundle sequence that the canonical embedding of $X$ satisfies $\Gamma(N(-2))=0$. (a) follows easily from (1.9) and (2.3.2). Further, $\kappa>d_{n-r-1}$ implies

$$
H^{1}\left(\Theta_{X}(-\kappa)\right) \cong \Gamma\left(\mathcal{O}_{X}(d-\kappa)\right)
$$

With $Y=Y_{n-r}$ as above, this last term equals $h^{0}\left(Y,-K_{Y}\right)$, and one easily finds that $H^{0}\left(\Theta_{Y}\left(-\left(K_{Y}+X\right)\right) \otimes \mathcal{O}_{X}\right)=0$. Now apply Corollary 3.12 .

Proof of 5.5. If $d=2 n+2$, Proposition 4.2 applies; so it remains to show that $X$ is on a Calabi-Yau variety when $n+1<d<2 n+2$. Let $X^{\prime}$ be a smooth hypersurface of degree $d^{\prime}=2 n+2-d$ intersecting $X$ transversally. Let $Z \rightarrow \mathbb{P}^{n}$ be the blow-up along $X \cap X^{\prime}$, with $\bar{X}$ and $\bar{X}^{\prime}$ the proper transforms; take the double cover $Y \rightarrow Z$ branched along $\bar{X} \cup \bar{X}^{\prime}$. Then $Y$ is a Calabi-Yau variety, with a divisor $\tilde{X}$ isomorphic to $X$, for which the normal bundle is $K_{X}$. In particular, $\mathcal{O}_{Y}(\tilde{X})$ is very ample in a neighborhood of $\tilde{X}$, and defines a morphism $Y \rightarrow Y^{*}$ which collapses $\tilde{X}^{\prime}$ to a rational singular point (since $d^{\prime}<n+1$ ). We can view $X$ as a hyperplane section of the singular Calabi-Yau $Y^{*}$ (cf. Lemma 3.4).

Proof of 5.6. If $d \leq n+1$, by Bertini's Theorem the general hypersurface of degree $n+1$ containing $X$ is smooth, and is a Calabi-Yau. (b) follows easily from (5.4), since $\kappa=d^{\prime}+d-(n+1)$. The condition in (c) may be written $2 \kappa=d$, so (5.2) applies. 


\section{REFERENCES}

[BM] A. Beauville and J.-Y. Mérindol, Sections hyperplanes des surfaces K3, Duke Math. J. 55 (1987), 873-878.

[Br] J. Brawner, The Gaussian-Wahl map for trigonal curves, Proc. A.M.S. 123 (1995), 13571361.

[CHM] C. Ciliberto, J. Harris, and H.P. Miranda, On the surjectivity of the Wahl map, Duke Math. J. 57 (1988), 829-858.

[CM] C. Ciliberto and H.P. Miranda, On the Gaussian map for canonical curves of low genus, Duke Math. J. 61 (1990), 417-443.

[DM] J. Duflot and H.P. Miranda, The Gaussian map for rational ruled surfaces, Trans. A.M.S. 330 (1992), 447-459.

[D] P. Du Val, On rational surfaces whose prime sections are canonical curves, Proc. London Math. Soc. 35, series 2 (1933), 1-13.

[E] D.H.J. Epema, Surfaces with canonical hyperplane sections, CWI Tract, I, Stichtung Mathematisch Centrum, Amsterdam (1983), ix+105 pp.

[GL] M. Green and R. Lazarsfeld, Special divisors on curves on a K3 surface, Invent. Math. 89 (1987), 357-370.

[I] S.P. Inamdar, On syzygies of projective varieties, Pacific J. Math. 177 (1997), no. 1, 71-76.

[L] R. Lazarsfeld, Brill-Noether-Petri without degeneration, J. Diff. Geom. 23 (1986), 299-307.

[Lv] S. L'vovsky, Extensions of projective varieties and deformations, I, II, Mich. Math. J. 39 (1992), 41-70.

$[\mathrm{MoM}] \mathrm{S}$. Mori and S. Mukai, The uniruledness of the moduli spaces of curves of genus 11, in Lecture Notes in Mathematics 1016, Springer-Verlag (1983), 334-353.

[M1] S. Mukai, Curves, K-3 surfaces, and Fano 3-folds of genus 10, in Algebraic Geometry and Commutative Algebra in Honour of M. Nagata (1987), 357-377.

[M2] S. Mukai, New development of theory of Fano 3-folds: vector bundle method and moduli problem, to appear, available at http://www.math.nagoya-u.ac.jp.

$[\mathrm{P}] \quad$ H. Pinkham, Deformations of algebraic varieties with $\mathbb{G}_{m}$ action, Astérisque 20 (1974), 1-131.

[Re] M. Reid, Special linear systems on curves on a K3 surface, J. London Math. Soc. (2) 13 (1976), 454-458.

[S] F.-O. Schreyer, Syzygies of canonical curves and special linear series, Math. Ann. 275 (1986), $105-137$.

[T] S. Tendian, Surfaces of degree d with sectional genus $g$ in $\mathbb{P}^{d+1-g}$ and deformations of cones, Duke Math. J. 65 (1992), 157-185.

[V] C. Voisin, Courbes tetragonals et cohomologie de Koszul, J. Reine Angew. Math. 387 (1988), 111-121.

[W1] J. Wahl, The Jacobian algebra of a graded Gorenstein surface singularity, Duke Math. J. 55 (1987), 843-871.

[W2] J. Wahl, On cohomology of the square of an ideal sheaf, J. of Alg. Geom. 6 (1997), 481-511.

Department of Mathematics, University of North Carolina, Chapel Hill, NC 27599-3250

E-mail address: jw@math.unc.edu 